\title{
MOLD OSCILLATION NEW TECHNOLOGY IND. 4.0 IN THE HIGH-SPEED CONTINUOUS CASTING FOR AHSS STEEL SLABS
}

\author{
Paul OLARU \\ EU - Expert, SCO Consulting GmbH, Luzern, Switzerland, polaru70@yahoo.com
}

https://doi.org/10.37904/metal.2019.666

\begin{abstract}
The use of technological packages and consequently following our technologic \& continuous casting principle, we achieve the shortest start-up times in the industry 4.0. Our modular solution approach ideally meets all technological requirements and customer demands, for both new and existing casters and for the production of carbon, stainless and special AHSS (Advanced High-Strength Steels) steel grades. The ever-greater demands for quality and the ongoing development of steel grades mean that the quality level at every stage of the casting process will continue to grow in importance in the future. As our example the CastFlex Oscillation (CFO) technology represents in 2019, the latest generation of Ind. 4.0, oscillation systems and saves money in operation compared to other systems [1]. The hydraulic oscillation drive system enables the dynamic adjustment of frequency, stroke, and wave form during casting for optimized negative strip time. The wear-free leaf-spring oscillator guidance system guarantees the highest guidance accuracy, outstanding operational safety, and low maintenance costs. The design allows a quick coupling of all media supplies to the mold and automatically completes the alignment of the mold and oscillator. By our metallurgical experience and technical expertise to supply cutting-edge technology for maximized bloom caster performance, production and upgrading.
\end{abstract}

Keywords: AHSS, continous casting, oscillation, industry 4.0, tundish

\section{INTRODUCTION}

The EU plant expansion began in late 2016 with melt-shop improvements, which included the commissioning of a new AHSS (Advanced High-Strength Steels) larger-section (210-mm square), four-strand continuous caster equipped with the latest online automated process controls to provide high-quality blooms [2]. The larger section gives the final product a higher reduction ratio, which greatly enhances overall internal quality. The new caster CastFlex Oscillation (CFO) technology represents in 2019, the latest generation of Ind. 4.0, oscillation systems also features a hydraulic oscillator, which allows oscillation to be adjusted to fit the characteristic needs of each unique grade of steel being produced. The line includes automated slag carryover detection and control from ladle to tundish coupled with a larger tundish for added flotation time. Argon shrouding and a one-piece submerged ceramic nozzle from tundish to mold prevent reoxidation during casting and enhance steel cleanliness. Closed-loop flow control from tundish to mold and automatic mold-powder monitoring and additions also improve surface quality. Advancements continued into 2018 with the commissioning of a new twin-tank vacuum degasser to support Monroe's high-production environment [3]. This gave the mill enhanced capabilities for lower-torr vacuum pressure and increased time under vacuum. In addition, the unit is equipped with automated cored alloy-wire feeding. This advanced equipment and capability results in exceptional cleanliness and precise chemistry control. In 2018, a new walking-beam reheat furnace and six additional rolling-mill stands were installed to process the larger $210-\mathrm{mm}$ blooms. The walking-beam furnace improves surface quality and lowers decarburization through better temperature and soaking-time control. A new pass design was developed to optimize reduction between stands in an oval to round configuration for a gradual reduction at each stand. This greater reduction ratio of rolled bars results in improved surface- and internal-quality characteristics. As a complement to the enhancements made in the melt shop and rolling mill, the latest technology Ind. 4.0, for value-added straightening and inspection capabilities was added in a new facility, which was designed to optimize AHSS process layout and product 
flow. The facility houses a new 10-roll high-speed automatic straightened and NDT capabilities, including ultrasonic testing with phased-array technology and magnetic-flux-leakage surface inspection and $100 \%$ inline X-ray grade verification, allowing for greater discernment of quality attributes. All of these equipment and process enhancements result in higher-quality engineered steel bars that support the forgers' need to meet the challenging performance expectations of the end users. These improvements also expand the array of applications that can be supported by forged SBQ steels.

\subsection{Advancements in Steel Cleanliness}

The global push for energy efficiency and stricter $\mathrm{CO} / \mathrm{CO}_{2}$ emission regulations has significantly increased the importance of clean steels in the automotive industry. The need for high-strength steel parts with lightweight design and increased fatigue life and performance is more important than ever. Bearing steels used in safetycritical parts - including wheel hubs, constant-velocity joints (CVJs), transmission and differential gears, steering racks and pinions, and others - are clear examples. Steel micro-cleanliness is critical to meet these needs and requires strict adherence to reliable meltshop practices. The EU plant team of R\&D, process and product metallurgists has made significant strides in sustainable steel cleanliness through enhanced meltpractice developments and strict process controls to produce bearing-quality steels in its continuous caster. Improved clean-steel practices have been developed to reduce the size and frequency of inclusions. These improvements have been validated using the latest automated SEM, extreme value analysis (EVA) and ultrasonic immersion testing equipment with low-, medium- and high-frequency capability. In addition, constant development of optimal refractories and fluxes are required to enhance clean-steel capabilities. The latest generation of wheel hubs and spindles is a perfect example. This highly stressed application has evolved over the last few years into today's wheel-bearing assembly. The EU plant design unit, which is permanently joined during the manufacturing process, consists of a medium-carbon steel spindle and outer ring. Any surface or subsurface nonmetallic inclusions could cause spalling and eventual failure. This occurs because the localized stresses are sufficient to cause micro-cracking around the inclusion, which propagates into larger, more severe cracking and eventually results in a spall failure. Modern clean-steel technologies Ind.4.0, mitigate these risks High levels of contact stress make the gear teeth susceptible to premature failure. The EU plant has engineered special AHSS grades focusing on higher cleanliness while maintaining the fine grain structure. These grades save time and costs in heat treating while providing higher fatigue life to the gears.

\section{EXPERIMENTAL TECHNOLOGY AND RESULTS}

Figures 1 shows a schematic of the molten steel in a continuous casting mold. The mold is cooled by water flowing through the grooves and acts as a heat sink. The steel solidifies against the copper mold and increases in thickness as it moves down the length of the mold. The steel shell is about $12 \mathrm{~mm}$ in thickness when it exits the mold. The mold is oscillated to prevent the sticking of the steel shell to the copper mold, and this oscillation promotes the infiltration of a film of liquid flux between the shell and the mold. Furthermore, the liquid mold flux on top of the liquid steel solidifies where it contacts the copper mold and gradually builds up a flux/slag rim. It has been theorized that the oscillation of the copper mold is responsible for the formation of oscillation marks. A mold simulator provides an ideal laboratory system for the study of initial solidification of steel in a continuous casting mold. The depth and width of the oscillation marks can be easily modified by changing the mold oscillation cycle, oscillation stroke, and casting speed. In addition, the effects of different mold fluxes on the initial heat transfer can be studied easily without interrupting the normal production operations at the plant.

A mold simulator provides an ideal laboratory system for the study of initial solidification of steel in a continuous casting mold. The depth and width of the oscillation marks can be easily modified by changing the mold oscillation cycle, oscillation stroke, and casting speed. In addition, the effects of different mold fluxes on the initial heat transfer can be studied easily without interrupting the normal production operations at the plant. 
The mold simulator developed in this study is an inverse type mold, where the steel solidifies around the mold, instead of the mold surrounding the solidifying steel.

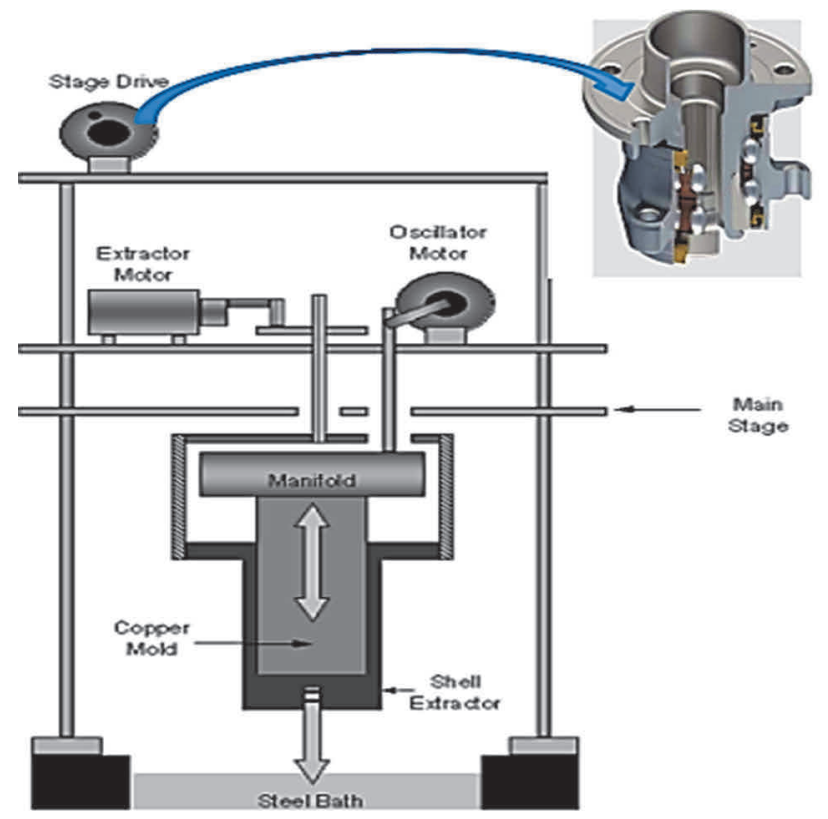

Figure 1 Schematic sketch of the mold simulator stage

Figure 2 is a schematic sketch of the mold simulator stage, which consists of several distinct modules to simulate the casting process [4]. The different physical modules of the simulator include the mold assembly, the extraction mechanism, the stabilization system, and the oscillation mechanism [5]. The mold assembly consists of a pair of grooved copper plates and a stainless steel baffle that separates the inlet and outlet water, as shown in Figure 2. In this work, the mold surface is flat instead of cylindrical, and is constructed from actual mold plates previously used at the EU Plant Steel. This flat plate configuration has nickel plating on the hot face, and the cold face is grooved with cooling channels. The cooling water is fed into the mold from the cooling water manifold, as shown in Figure 2. In order to simulate continuous casting, the mold assembly is fitted with an extraction mechanism, which is fabricated from 6.25-mm-thick steel plates. The extractor pulls the solidifying steel shell in the casting direction (downwards). This exposes liquid steel to the water-cooled copper mold at the meniscus and allows the formation of a new steel shell. The extractor is designed so that only one face. Also shown in Figure 3, the physical locations of the thermocouples with respect to the meniscus (see Figure 2 write side). These thermocouples are located 133, 140, 146, 152, 159, and $165 \mathrm{~mm}$ from the bottom of the mold. The

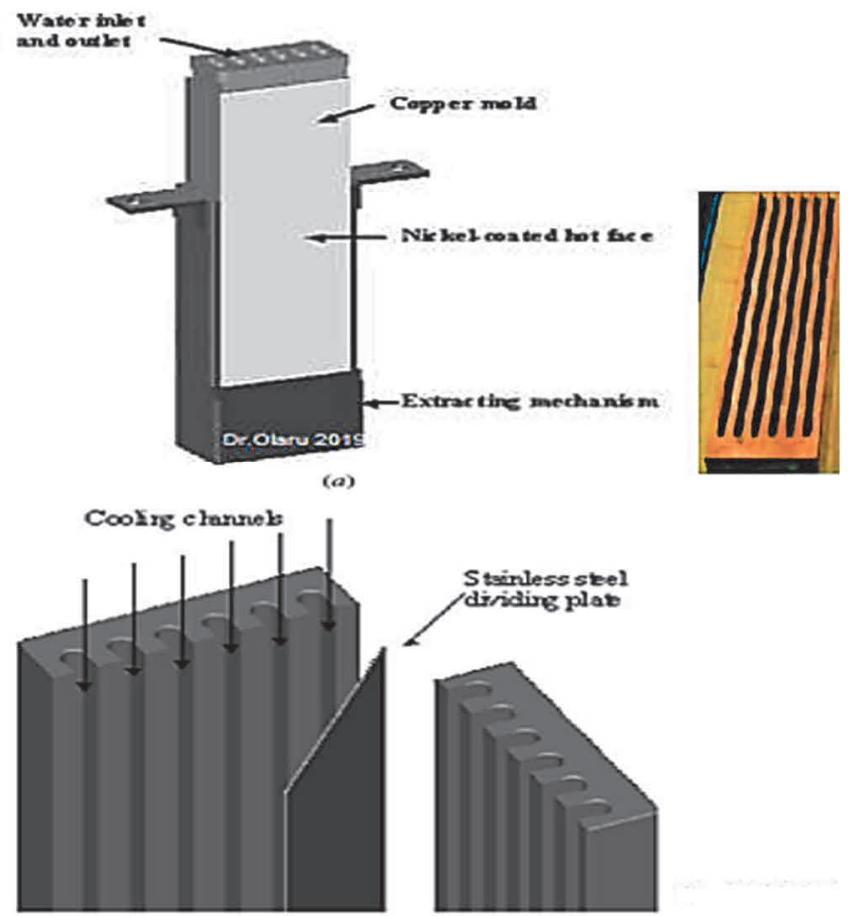

Figure 2 The mold copper assembly and extractor mechanism 
molds were machined with 1.59-mm-diameter 50-mm-deep thermocouple voids with an orientation parallel to the mold surface to install the thermocouples. So that the positions of the tips were well defined, and the tips were covered with a heat sink compound to enhance heat transfer to the thermocouple tip. um oxide insulation and a stainless steel sheath with an outer diameter of $0.5 \mathrm{~mm}$. The thermocouples wereln addition to the thermocouples, linear velocity displacement transducers (LVDTs) were used to monitor the motions of the mold and the extractor mechanism [6]. Our Lab. software was used to acquire temperature data at $60 \mathrm{~Hz}$ for the duration of the experiment to detect phenomena occurring within individual oscillation cycles. The temperature data acquired from the mold thermocouples were used to develop an estimate of the heat flux through the mold during the initial solidification of the steel shell using the one-dimensional inverse heat conduction program developed by my technology.

In the mold simulator experiments, the known boundary condition is the convective cooling of the mold by water flowing through cooling channels. While there are correlations for determining the heat-transfer coefficient for the cooling channels, another thermocouple (Figure 3), was introduced into the domain to provide a well-defined boundary condition. In this case, the first thermocouple was $1.5 \mathrm{~mm}$ away from the heated surface, while the second thermocouple $5.0 \mathrm{~mm}$ away from the heated surface was used to define the subdomain.

\section{Sco Consulting}

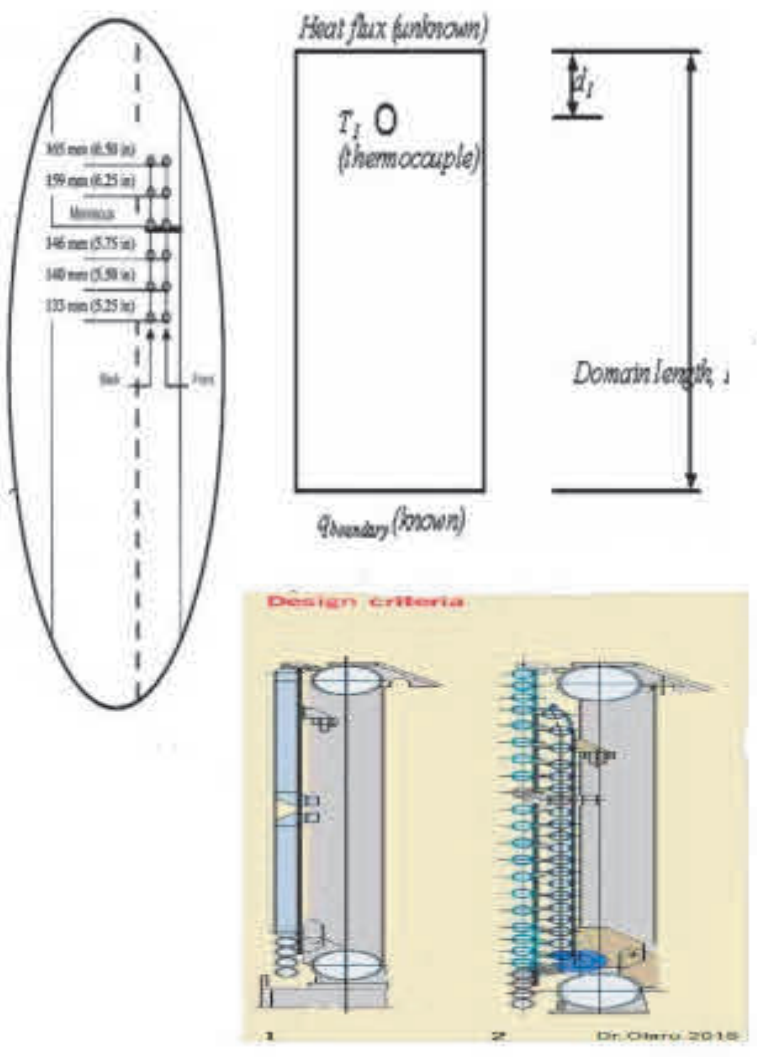

Figure 3 The thermocuples located for description of thermic fluxes

\section{CONCLUSIONS}

Protected from reoxidation, the liquid steel is poured from a conventional ladle into a covered tundish and from there, via the submerged entry nozzle, into the preheated feeding system. From this system the steel flows onto the moving horizontal mold which consists of the conveyor belt and internally cooled side dams. The 
conveyor belt is intensively water cooled from the bottom. No oscillating mold is therefore needed for initial solidification; no addition of casting flux is required. Above the conveyor belt an inert gas atmosphere protects the solidifying steel. At the rear end of the caster, a defined gas mixture directly influences the solidification structure. Electro-magnetic units to influence the steel flow as well as cooled hoods are arranged above the conveyor belt. A transverse stirrer supports the smooth and uniform distribution of the liquid steel up to the side dams. A longitudinal stirrer synchronizes the movement of the conveyor belt with the liquid steel flow. This stirring technology is the result of a joint development. CastFlex Oscillation (CFO) technology delivered both units. The as-cast strip leaves the conveyor belt horizontally. It is guided by a top roller and three pairs of ironing rollers that can influence the flatness of the strip. Downstream these rollers both the strip thickness and profile are measured. The as-cast strip then enters an enclosed roller table in which the complete inertization of the process is ensured up to the end of the table. The results achieved up to today are quite promising. The process reproducibility and the plant availability have been continuously enhanced. Satisfactory answers were obtained on process-related basic questions concerning wear, the conveyor belt and the moving side dams. This EU plant is designed for a maximum steel quantity of approx. $70 \mathrm{t}$ per cast. The next phase in the digitalisation of the manufacturing sector Continuous Casting for AHSS Steel Slabs, driven by four (4) disruptions:A-The astonishing rise in data volumes, computational power, and connectivity, especially new low-power wide-area networks;B-The emergence of analytics and business intelligence capabilities;C- New forms of human-machine interaction such as touch interfaces and augmented-reality systems;D-Improvements in transferring digital instructions to the physical world, such as advanced robotics and 3-D printing.

\section{REFERENCES}

[1] MILLS, K.C., KARAGADDE, S., LEE, P.D., YUAN, L. and SHAHBAZIAN, F. Calculation of physical properties for use in models of continuous casting process-part 1: mould slags. ISIJ Int. 2016. vol. 56, no. 2, pp. 264-273.

[2] SPRAGG, K., NOEPPEL, A., LACOMBE, J.K., DUMOND, M., ERNST, R. and DELANNOY, Y. Induction processing of liquid materials. In 16th Int. Conf. UIE2008, Krakow, 2008.

[3] DUMOND, M., ERNST, R., GARNIER, C. and PETITPAS, P. Recent progresses in optimizing inductive cold crucible processes. In 6th Int. Conf. on Electromagnetic Processing of Materials, EPM, Dresden, 2009.

[4] DUMOND, M., ERNST, R., GARNIER, C., GATHFAN, H. and PETITPAS, P. Innovative Inductive Cold Crucible Configurations with improved Efficiency. J. of Iron and Steel Research Int. 2012. vol. 19, pp. 669-672.

[5] SPITANS, S., BAAKE, E., JAKOVICS, A. and FRANZ, H. Numerical Simulation of EM levitation in cold crucible furnace. Magnetodydrodynamics. 2015. vol. 51, no.3, pp. 567-578.

[6] BOJAREVICS, V., HARDING, R. A. and PERICLEOUS, K. The development and validation of a numerical model of an induction skull melting furnace. Metallurgical and Materials Transactions. 2004. vol. 35 B, pp. 785-803. 